\title{
Iglesias. Archéologie des entreprises minières
}

\section{Nicolas Minvielle Larousse}

\section{OpenEdition \\ Journals}

Édition électronique

URL : https://journals.openedition.org/baefe/4224

DOI : $10.4000 /$ baefe.4224

ISSN : 2732-687X

Éditeur

ResEFE

\section{Référence électronique}

Nicolas Minvielle Larousse, «Iglesias. Archéologie des entreprises minières » [notice archéologique], Bulletin archéologique des Écoles françaises à l'étranger [En ligne], Italie, mis en ligne le 14 septembre 2021, consulté le 16 septembre 2021. URL : http://journals.openedition.org/baefe/4224 ; DOI : https:// doi.org/10.4000/baefe.4224

Ce document a été généré automatiquement le 16 septembre 2021.

\section{cc) (†) $\odot$}

Le Bulletin archéologique des Écoles françaises à l'étranger est mise à disposition selon les termes de la Licence Creative Commons Attribution - Pas d'Utilisation Commerciale - Pas de Modification 4.0 International. 


\title{
Iglesias. Archéologie des entreprises minières
}

\author{
Nicolas Minvielle Larousse
}

\section{NOTE DE L'AUTEUR}

Date précise de l'opération : 01/03/2021 au 28/03/2021

Composition de l'équipe de terrain : Nicolas Minvielle Larousse, Guillaume de Meritens de Villeneuve.

Établissements porteurs de l'opération : EFR

Remerciements : La surintendance de la province du sud Sardaigne, Maura Picciau, Sabrina Cisci ; le Distretto minerario della Sardegna, Giorgio Paolucci ; l'Archivio Storico Minerario, Alessandro Cuccu, Valentina Venne, Franco Plinio Vacca, Maurizio Milia ; le Spéléo-Club de Domusnovas, Angelo Naseddu ; ainsi que Gianpaolo Atzei, Mauro Buosi, Francesca Sanna, Guillaume de Meritens de Villeneuve, Benoit Favennec, Élodie Paris.

\section{Introduction}

1 La Sardaigne est une « île aux filons d'argent » exploitée pendant plusieurs millénaires, de l'Antiquité à la fin du XX $\mathrm{X}^{\mathrm{e}}$ siècle ${ }^{1}$. Abondamment repris entre les XII ${ }^{\mathrm{e}}$ et XIV $\mathrm{X}^{\mathrm{e}}$ siècles, les gisements polymétalliques (plomb, argent) qui se concentraient dans les montagnes paléozoïques d'Iglesias firent de l'île l'une des principales argentières de la Méditerranée ${ }^{2}$. C'est ce terrain industriel, marqué par les entreprises minières, qui fait l'objet d'une enquête archéologique, cela dans les cadres géographiques fixés par les sources écrites médiévales. Ce bref compte-rendu est celui d'une première campagne de prospection effectuée au printemps 2021, avec comme objectif d'identifier et de reconnaître les secteurs qui ont fait l'objet de travaux anciens ${ }^{3}$.

2 La prospection a été précédée par le dépouillement d'une partie des archives contemporaines, car elles mentionnent régulièrement les vestiges d'exploitations 
antérieures aux reprises des XIX et $\mathrm{XX}^{\mathrm{e}}$ siècles ${ }^{4}$. Pour parvenir à un premier inventaire représentatif des lieux où se trouvent ces travaux et avoir un aperçu de leurs formes, les plans établis lors des prises de concession au XIX ${ }^{\mathrm{e}}$ siècle, puis ceux établis à mesure de l'avancée des travaux ont d'abord été mis à contribution. Plus de 3000 plans et coupes concernant la Sardaigne en général et l'Iglesiente en particulier ont été dépouillés, les 58 les plus pertinents ont été géoréférencés et les 3211 vestiges qu'ils représentaient numérisés. Le fichier topographique créé a été la principale fondation des prospections ${ }^{5}$. Par ailleurs, la région de Sardaigne a mis en ligne des MNT au pas de $1 \mathrm{~m}$ issus de relevés LIDAR. Toute lîle n'est pas concernée, mais les littoraux, les abords de fleuves et certaines zones critiques sont couverts. L'Iglesiente est majoritairement intégrée, à l'exception de quelques vides situés notamment au centre et au sud. Ces relevés ont aussi servi de guide aux prospections et de support aux relevés de terrain.

De brèves reconnaissances pédestres ont été réalisées dans chacune des 14 zones suggérées par la représentation de travaux anciens sur les plans des concessions minières des $\mathrm{XIX}^{\mathrm{e}}$ et $\mathrm{XX}^{\mathrm{e}}$ siècles - à l'exception de 4 zones, 3 en raison de l'ampleur des reprises contemporaines, 1 par manque de temps ${ }^{6}$. Ces zones ont fait l'objet de plusieurs visites extensives, en fonction de leur étendue (fig. 1). Les secteurs qui avaient particulièrement concentré les reprises contemporaines ont pour le moment été exclus. Ces reconnaissances avaient deux objectifs : 1/visualiser en général le terrain, l'état des vestiges, l'impact des reprises contemporaines, identifier des épandages de mobilier sans toutefois faire de ramassage; 2/ effectuer des relevés photogrammétriques par drone lorsque la végétation le permettait, afin d'identifier plus efficacement les éléments. Les vestiges reconnus ont été ajoutés au fichier commencé avec le LIDAR. Il compte à ce jour 730 occurrences 7 .

Fig. 1. Implantation 2021 des prospections pédestres et des relevés aériens.

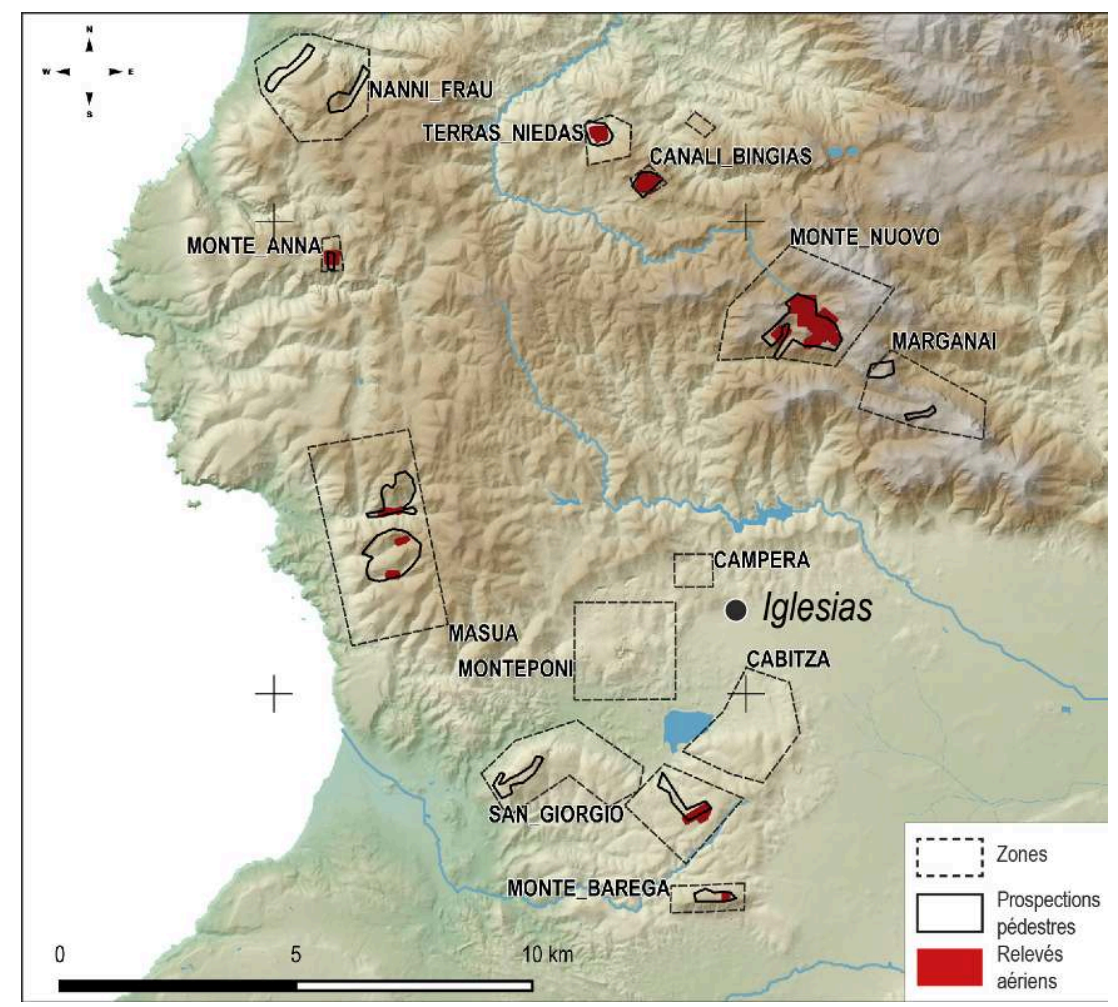




\section{Résultats 2021}

Les résultats ci-dessous sont présentés par zones, de façon analytique. L'ensemble des informations livrées ici ne constitue qu'un état de la recherche, résumé, issu du rapport d'opération remis à la surintendance de la province du Sud Sardaigne ${ }^{8}$.

\section{Canali Bingias (zone 3)}

5 Les mines de Canali Bingias se trouvent sur le versant septentrional de la vallée d'Antas, à $850 \mathrm{~m}$ au nord-est du temple. Les vestiges visibles sont variés : on trouve d'une part des travaux miniers, parfois associés à des haldes, et d'autre part un ensemble de structures en ruine. L'essentiel se concentre sur le plateau, mais une halde et l'entrée d'une galerie se situent à environ $200 \mathrm{~m}$ en aval, le long du chemin d'accès. Les travaux miniers les plus imposants sont six fosses verticales de 10 à $30 \mathrm{~m}$ de côté et de plusieurs dizaines de mètres de profondeur (fig. 2). Leurs bords comme leurs parois sont irréguliers et sont partiellement comblés par des arbres. Autour de ces fosses, on observe une dizaine de puits de 1 à $2 \mathrm{~m}$ de diamètre - certains isolés, d'autres regroupés. Enfin, quelques tranchées et renfoncements sont visibles sans qu'il soit possible en l'état de discerner s'il s'agit d'ébauches d'ouvrages miniers ou de travaux comblés. La galerie qui s'ouvre en aval est vraisemblablement un travers-banc destiné à atteindre les gisements du plateau par le bas. Les haldes qui parsèment le plateau, dispersées entre les fosses et les puits, semblent provenir de rejets d'extraction, car elles sont essentiellement constituées de calcaires gris. Les structures visibles se composent, au nord, d'un bâtiment de $23 \mathrm{~m}$ de long et de $10 \mathrm{~m}$ de large, compartimenté au sud par 5 pièces; de deux fondations massives et circulaires de 13,5 $\mathrm{m}$ de diamètre, situées à proximité de profondes fosses et d'au moins quatre petits bâtiments quadrangulaires ruinés, situés aux quatre coins de l'espace exploité. À l'exception du bâtiment compartimenté, construit avec un liant, recouvert d'enduits et d'un toit de tuiles, les autres structures semblent construites en pierres sèches.

Fig. 2. Orthophoto d'une fosse minière (Canali Bingias).

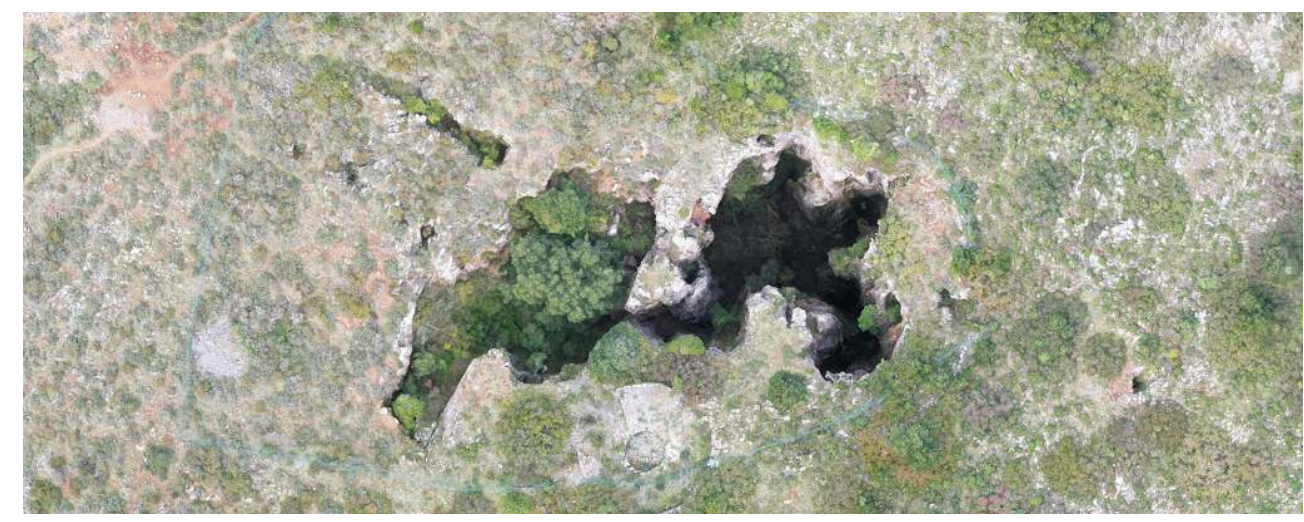

6 À Canali Bingias, des phases antiques - punique et romaine - sont présumées en lien avec l'occupation de la vallée d'Antas. Il manque cependant une confirmation, documentaire ou archéologique. Dans le même ordre d'idée, une phase médiévale serait aussi possible, en lien avec le territoire de la villa d'Antasa, cité avec des mines dans le 
Bref d'Iglesias. L'exploitation a été reprise au XIX siècle. Un long bâtiment compartimenté accompagnait vraisemblablement cette phase de l'exploitation les treuils en pierre construits à proximité des fosses. Il n'est pas encore possible en revanche de proposer une attribution chronologique pour les autres structures quadrangulaires.

\section{Marganai (zone 5)}

7 Les mines se localisent sur les crêtes orientales de la vallée du Canali Aquas. La majeure partie des travaux miniers sont des lignes de puits verticaux de 0,5 à $1,5 \mathrm{~m}$ de diamètre. Creusés dans le calcaire gris ou les dolomies, ils suivent des failles et leurs bords sont irréguliers. On remarque aussi des travaux plus longiformes, en bord de falaise notamment. Une large fosse minière est remarquable: de $100 \mathrm{~m}$ de long et $50 \mathrm{~m}$ de large. Ses alentours ont néanmoins été réaménagés (bâtiments, héliport) et l'intérieur de la fosse est recouvert par la forêt. Enfin, des haldes sont visibles sur les vues aériennes de 1954 et 1968, aujourd'hui disparues à cause des aménagements et de la végétation.

8 Une phase antique - romaine - est présumée au Marganai. Des objets auraient été mis au jour dans les réseaux ou les environs (information orale à confirmer). De même, des reprises médiévales sont possibles, car le massif du Marganai pourrait s'identifier au Monte di Pietra Carfita cité dans le Bref d'Iglesias. Les reprises contemporaines, à partir de la seconde moitié du XIX ${ }^{e}$ siècle, sont à l'origine du percement de plusieurs galeries de rabais destinées à exploiter les parties basses des gisements.

\section{Masua (zone 6)}

9 Les mines de Masua constituent un large ensemble allant du Monte Narba au nord au Monte San Giuseppe au sud. Autour de Masua et de Nebida, le paysage est profondément marqué par les activités minières contemporaines: extraction en surface et en souterrain sur les montagnes et les falaises, traitement des minerais en contrebas, le long du littoral. Ce paysage conserve cependant les traces de travaux anciens commencés à ciel ouvert, par fosse ou par puits. Les vallées qui entourent la Punta Rubicina et la vallée de Matoppa concentrent les vestiges les plus évident et a priori les moins repris. Dans la vallée de la Rubicina, six fosses d'une dizaine de mètres de diamètre sont figurées, accompagnées de 8 puits et de trois structures. La vue aérienne de 1968 fait distinctement apparaître certains de ces travaux accompagnés de haldes. Sur place, les travaux miniers sont en partie comblés et recouverts par la végétation. Plus en amont dans cette vallée, un autre groupe de travaux est visible sur son versant nord : plusieurs puits accompagnés de haldes. La vallée méridionale, Cuccu Aspu, comprend plusieurs fosses ou puits partiellement comblés, en particulier à la base de la colline de Sa Pruixina. Tout au long du col de Cuccu Aspu s'étirent des travaux miniers à ciel ouvert, en tranchée, puits ou fosse, accompagnés de haldes. La vallée de Matoppa, située plus au nord en contrebas du Monte Narba, présente des vestiges similaires : au moins trois puits accompagnés encore aujourd'hui de haldes. Si le puits occidental est comblé, on observe une plate-forme de $20 \mathrm{~m}$ de côté aménagée dans le versant de la pente. Sa halde se développe en contrebas. 
10 À Masua, des phases de production protohistoriques sont présumées, en lien avec la découverte de lampes lors des reprises minières contemporaines, mais restent à confirmer9. Aucun élément connu n'est attaché à la période antique. Des travaux médiévaux sont en revanche plus probablement présents. Le Monte di Malva, cité dans le Bref, pourrait s'identifier au Monte Narba. Sur le terrain, les vestiges observés sont trop comblés ou végétalisés pour confirmer ou affiner la chronologie.

\section{Monte Anna (zone 7)}

11 Les mines se localisent $3 \mathrm{~km}$ au sud-est de Buggeru, sur le versant méridional du Monte Anna, entre la Punta Baxia et le Monte Basileddu. Deux types d'ouvrages sont visibles: des travaux à ciel ouvert situés sur le versant du Monte Anna, et des travaux en galerie qui s'ouvrent à sa base. Parmi les premiers se mêlent des puits et des tranchées superficiels ou comblés. Les dimensions sont modestes, autour du mètre de côté. L'un d'eux en revanche est une fosse de 15 et $20 \mathrm{~m}$ de côté, profonde d'au moins une trentaine de mètres. Dans tous les cas, les bords sont irréguliers. Les galeries s'ouvrent quant à elles sur les flancs orientaux (au moins 2) et méridionaux (au moins 4) du versant et sont accompagnées de haldes. Signalons enfin une cavité située dans le coin sud-ouest du versant, non approchée lors des reconnaissances, mais visible sur le relevé aérien.

12 Aucun indice chronologique, documentaire ou archéologique, n'a encore été signalé au Monte Anna. Cela étant, le plan de détail permet une assez bonne discrimination entre les travaux modernes et anciens. Sur le versant du mont, parmi la vingtaine de travaux à ciel ouverts recensés, sept puits sont qualifiés d'antichi. Toutes les galeries en revanche seraient des ouvrages de rabais, destinés à atteindre les parties basses des gisements lors des reprises contemporaines.

\section{Monte Barega (zone 8)}

Le Monte Barega se situe au sud d'Iglesias et domine la vallée du Flumentepido. Il forme une crête s'élevant à $450 \mathrm{~m}$ d'altitude, d'environ $1 \mathrm{~km}$ de long et de $100 \mathrm{~m}$ de large. Une seule fosse a été à ce jour relevée. Elle entaille la roche sur $30 \mathrm{~m}$ de long et 10 de large, sur une profondeur d'au moins $5 \mathrm{~m}$. Elle est comblée, mais on observe une continuité qui rejoint deux autres fosses situées $25 \mathrm{~m}$ plus au nord. Signalons en complément que Marta Macri a identifié deux autres fosses à Sa Fossa Teula lors de ses prospections, des traces de rubéfaction, de la céramique médiévale ainsi que des tegulae, cela dans un rayon de $100 \mathrm{~m}$ autour des fosses ${ }^{10}$.

14 Aucun indice antique n'est à ce jour connu. Les mines de Barecha sont en revanche citées dans le Bref d'Iglesias. Les travaux anciens reconnus en ces lieux peuvent donc être attribués, au moins en partie à cette période. Les gisements de baryte du Monte Barega ont largement été exploités à partir de la seconde moitié du XIXe siècle et les environ de la fosse ont aussi été remaniés.

\section{Monte Nuovo (zone 9)}

15 Les travaux miniers du Monte Nuovo se répartissent sur un massif situé entre San Benedetto et la Malacalzetta, en rive droite du Canali Acquas. Il se compose de plusieurs 
collines, du Monte Perda à l'ouest au Montinou à l'est - duquel dérive le nom donné à cette zone. Le massif a été exploité de façon intense aux périodes anciennes et contemporaines. Il semble cependant, d'après les différents plans des concessions, que les lieux ayant fait l'objet des travaux les plus intenses dans les deux derniers siècles se concentrent à l'ouest (Monte Perda, Arcu Sa Cruxi) et au nord (vallée de la Malacalzetta), tandis que les lieux les plus exploités aux périodes antérieures se trouvent à l'est, aux alentours de la Punta Sa Meni et de Montinou (fig. 3).

Fig. 3. Plan des travaux anciens au Monte Nuovo, d'après les concessions $\mathrm{XIX}^{\mathrm{e}}$ et $\mathrm{XX}$ e siècles.

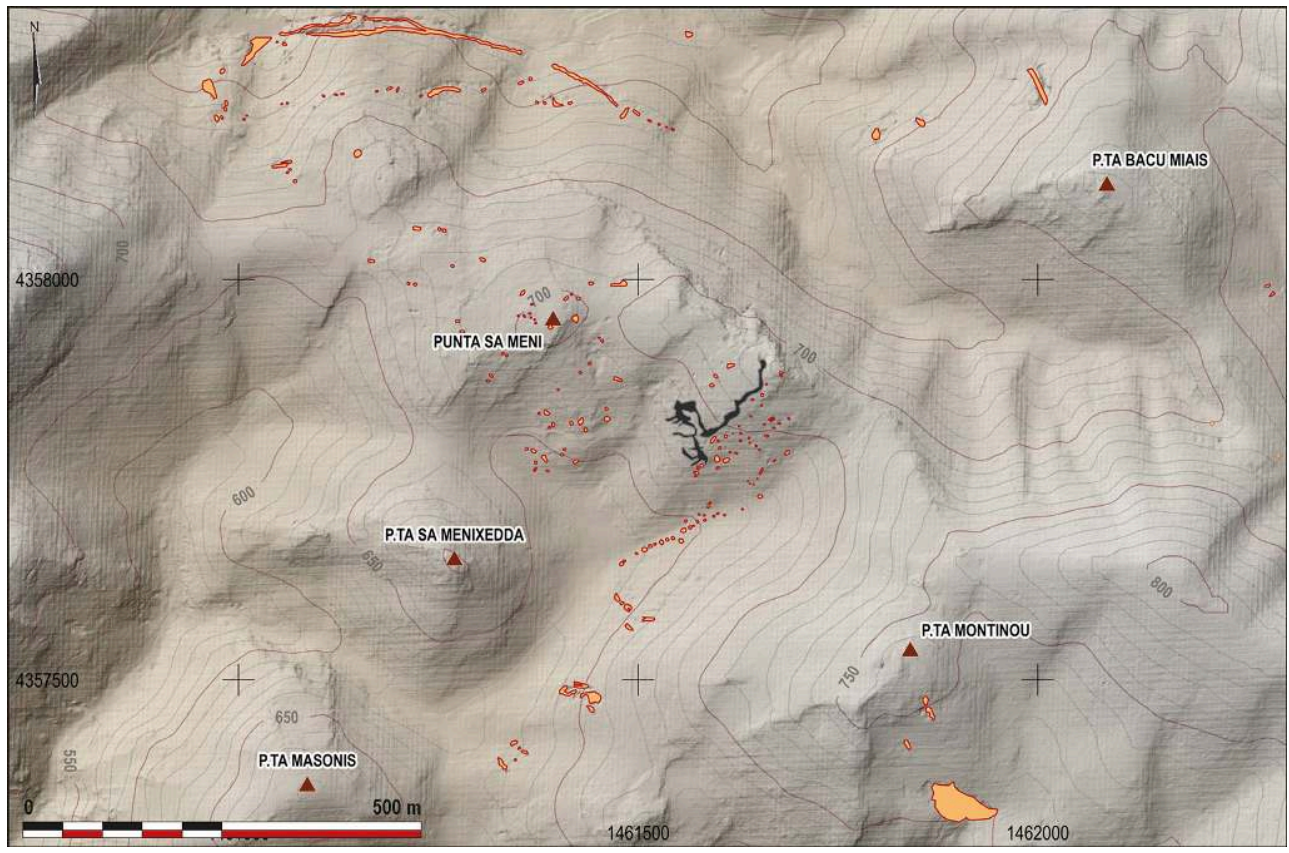

Le secteur de la Malacalzetta, au nord, est marqué par une étroite tranchée à ciel ouvert, complétée par une dizaine de petites fosses ou de puits dispersés plus en amont, ainsi que d'une grande halde de $150 \mathrm{~m}$ de long sur $40 \mathrm{~m}$ de large. Le secteur de la Punta sa Meni, à l'est, rassemble de nombreux travaux anciens qui se présentent sous la forme de puits de petites dimensions (souvent moins d'un mètre), de petites fosses (quelques mètres de côté) ou encore de grattages. Des travaux plus significatifs sont présents au sud-est. Un puits d'une dizaine de mètres de côté situé au sommet de la crête donne notamment accès à un chantier souterrain de $260 \mathrm{~m}$ de développement. Plusieurs haldes sont visibles en surface. Signalons enfin, à la base du versant, l'existence d'un long amas de blocs ( $60 \mathrm{~m}$ sur $20 \mathrm{~m}$ ) associé à des travaux de surface et quelques tessons de céramiques glaçurées. Le secteur de Montinou, au sud, rassemble un large puits de $6 \mathrm{~m}$ de côté accompagné d'une halde qui se développe en cône sur $20 \mathrm{~m}$ de long et $30 \mathrm{~m}$ de large (fig. 4). On trouve au moins trois puits situés sur son versant sud-est, accompagnés d'une légère halde. Une centaine de mètres plus au sud-est sur ce même versant s'ouvre une large fosse de $75 \mathrm{~m}$ de long sur $40 \mathrm{~m}$ de large. Profonde d'environ $5 \mathrm{~m}$ au maximum, elle est comblée. Trois groupes de travaux à ciel ouvert se trouvent à la base de son versant occidental. Le groupe nord est composé de travaux en tranchée, a priori superficiels. Deux haldes semblent être associées. Le groupe central est composé de travaux en tranchée approfondis, jusqu'à se transformer en fosses. En aval, trois murs de terrasses ont été successivement construits afin de ménager un espace plan. Le groupe sud se compose de 4 ouvrages au moins, dont deux larges puits 
de 3 à $5 \mathrm{~m}$ de côté. Signalons enfin la présence, $100 \mathrm{~m}$ plus en aval, des ruines d'une structure quadrangulaire située à proximité du chemin. Le secteur de la Punta Coremu, à l'ouest, comprend de longues et étroites tranchées à ciel ouvert -2 orientées nord-sud de 60 et $90 \mathrm{~m}$ de long, et 2 orientées est-ouest de 40 et $60 \mathrm{~m}$ de long.

Fig. 4. Vue 3D du puits du Montinou et de sa halde (Monte Nuovo).

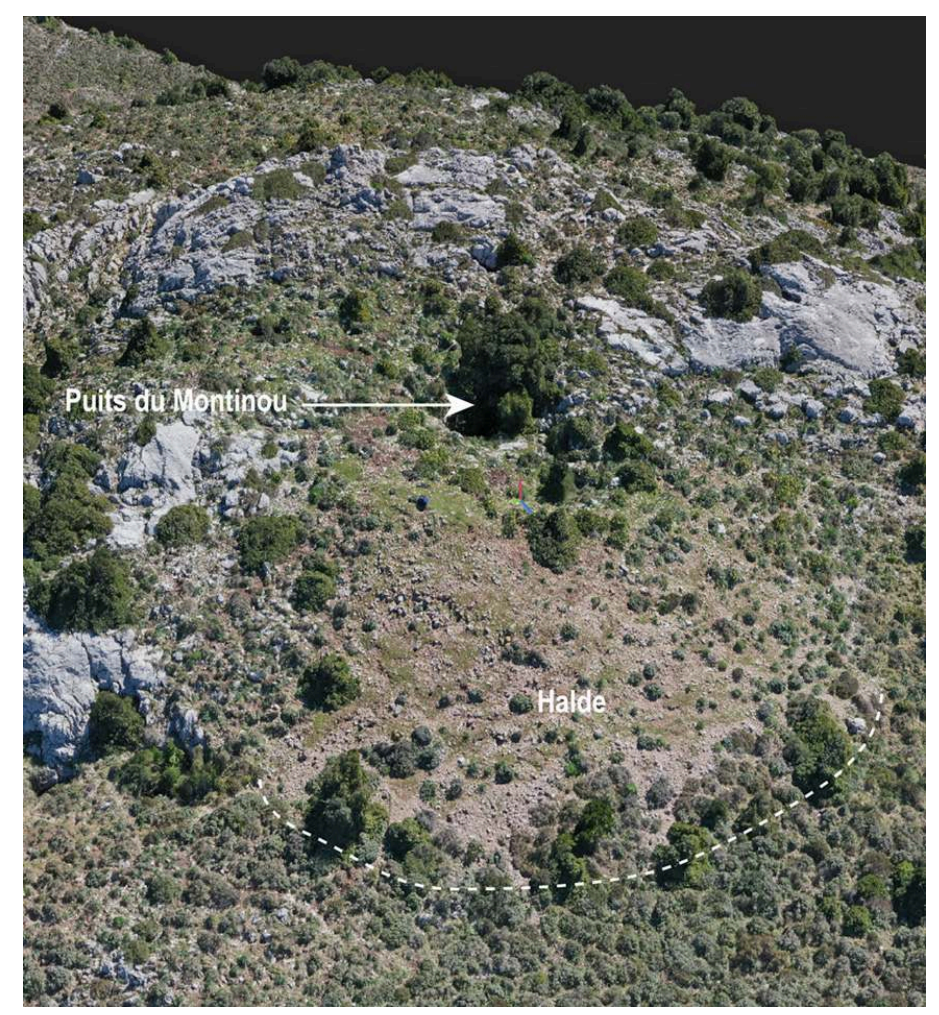

17 Aucun indice d'exploitation antique n'est pour le moment connu dans ce massif. En revanche, le Monte Nuovo est cité dans le Bref d'Iglesias comme l'une des montagnes exploitées. Si l'identification avec le Montinou est exacte, alors au moins une partie des travaux anciens observés dateraient du Moyen Âge central. Sur le terrain, l'ensemble des travaux à ciel ouverts observés présentent une forme et une facture qui peut effectivement les rattacher à une exploitation ancienne et on a observé plusieurs chantiers d'abattage qualifiés d'anciens par les plans et les coupes. Enfin, l'amas de blocs situé en contrebas de la Punta sa Meni contient du matériel céramique, tournée rouge ou grise et vernissée. Les reprises des XIX et XXe siècle correspondent surtout au percement de galeries de rabais, mais des prospections et des travaux de surface ne sont pas à exclure.

\section{Monte San Giovanni (zone 11)}

Le Monte San Giovanni est un massif situé entre Iglesias et Gonnesa. Il se compose de plusieurs collines, allant du Monte San Giovanni stricto sensu, à l'ouest, à la Punta Is Ollastus à l'est, avec une extension jusqu'à la Punta de Is Guardianus. Il s'agit de l'une des montagnes les plus exploitées de l'Iglesiente. Les travaux contemporains ont très largement recoupé et élargi les anciens travaux en surface, tout en atteignant la base des gisements par des ouvrages en galerie et des chantiers souterrains. Identifier les phases antérieures au XIX ${ }^{\mathrm{e}}$ siècle est donc un défi possible, mais de longue haleine. La 
confrontation des plans levés au XIX ${ }^{e}$ siècle avec le relevé LIDAR permet déjà d'identifier certains secteurs, mieux préservés que d'autres. Ainsi au sud de la Punta Is Ollastus, au nord de la Punta de Is Guardianus, et au sommet de la colline qui les sépare. La Grotta pisana est un bel exemple de ces vestiges conservés au milieu des reprises contemporaines ${ }^{11}$. Il s'agit d'un réseau minier souterrain long d'une centaine de mètres. Il conserve des ouvrages représentatifs des techniques de percement anciennes (outils, feu), des remplissages et des remblais contenus par des murs de soutènement. L'entrée de la mine est de plus associée aux vestiges d'un bâtiment quadrangulaire de $8 \mathrm{~m}$ de large sur $6 \mathrm{~m}$ au moins de long (fig. 5). Une citerne, creusée dans le rocher puis maçonnée à la chaux se trouve sur le bord sud-est du bâtiment. Enfin, signalons d'autres structures construites plus près encore de l'entrée de la mine. Ces dernières semblent toutefois plus tardives et pourraient avoir été installées après un élargissement à la poudre de cette entrée.

Fig. 5. Bâtiment à l'entrée de la Grotta pisana (Monte San Giovanni).

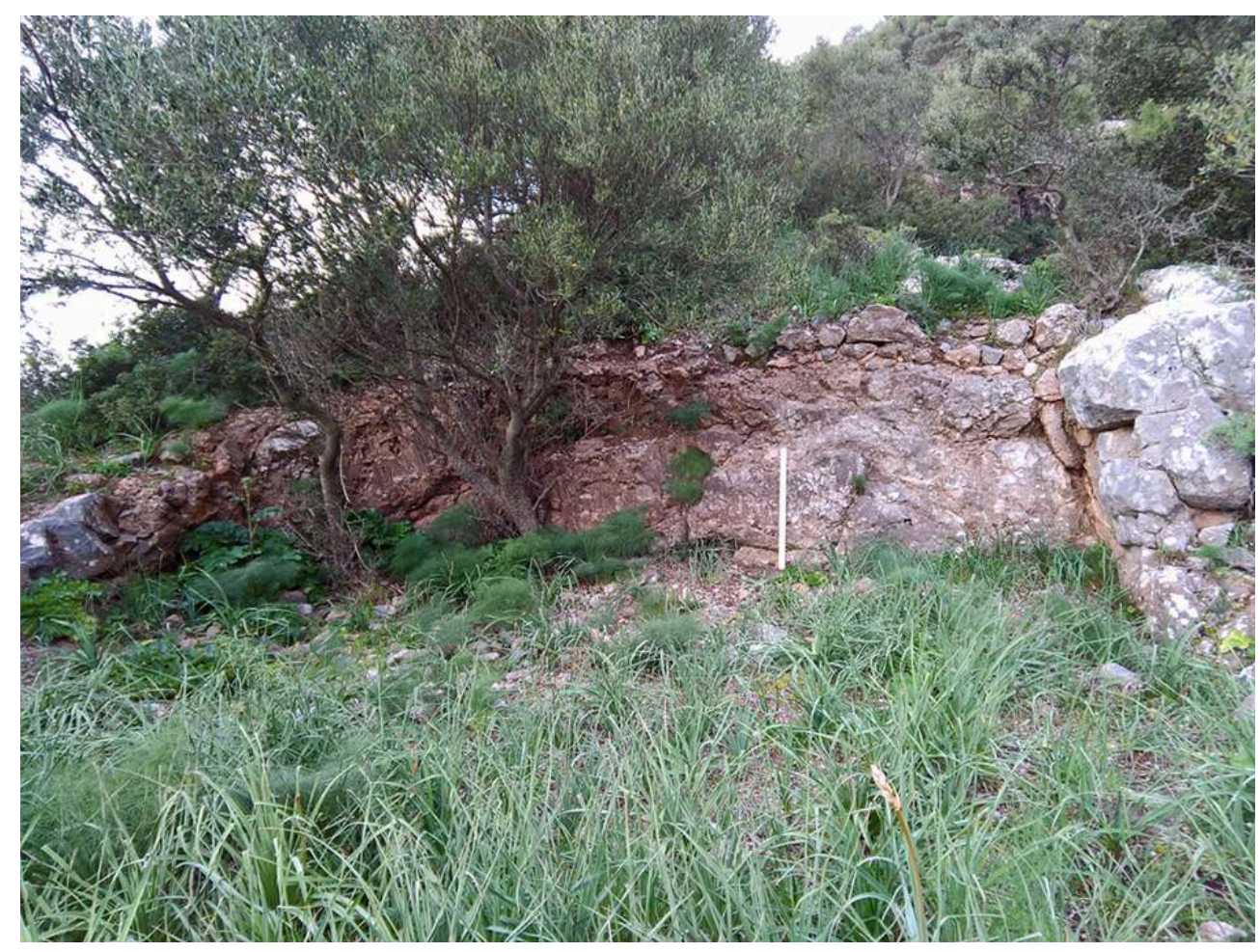

19 Aucun indice antique n'est actuellement connu au Monte San Giovanni. Il est en revanche cité à plusieurs reprises au début du XIVe siècle lorsqu'il est question de l'Argenteria de Conesa. Une partie des fosses et des travaux anciens sont donc attribuables à cette période. Les reprises des $\mathrm{XIX}^{\mathrm{e}}$ et $\mathrm{XX}^{\mathrm{e}}$ siècles sont perceptibles partout et se caractérisent notamment par l'élargissement des fosses en surface et le percement de réseaux en galerie, sous la falaise septentrionale.

\section{Nanni Frau (zone 12)}

20 Les mines de Nanni Frau se localisent autour d'un massif circulaire de $2 \mathrm{~km}$ de diamètre qui domine la plaine de San Nicolo. Les travaux miniers prennent majoritairement la forme de tranchées à ciel ouvert avec de possibles prolongements souterrains. Elles 
peuvent être larges - environ 5 à $10 \mathrm{~m}$ - ou étroites avec des largeurs de moins de 1 mètre. En deçà du Bacchis e Montis, on observe un groupe de puits situés en fond de vallée, puis une ligne de travaux qui s'étend sur le versant, en montant jusqu'au sommet de la crête. La prégnance de la végétation rend l'observation difficile, mais il semble que ces travaux aient été substantiels - larges tranchées comblées, prolongements souterrains présumés - et n'aient pas fait l'objet de reprises. Au-dessus de San Nicolo, les longues tranchées subverticales sont parfaitement visibles et fréquemment accompagnées de haldes (fig. 6). Les chantiers d'abattage se prolongent également en souterrain, toujours en suivant cet axe minéralisé.

Fig. 6. Vue d'une tranchée de San Nicolo (Nanni Frau).

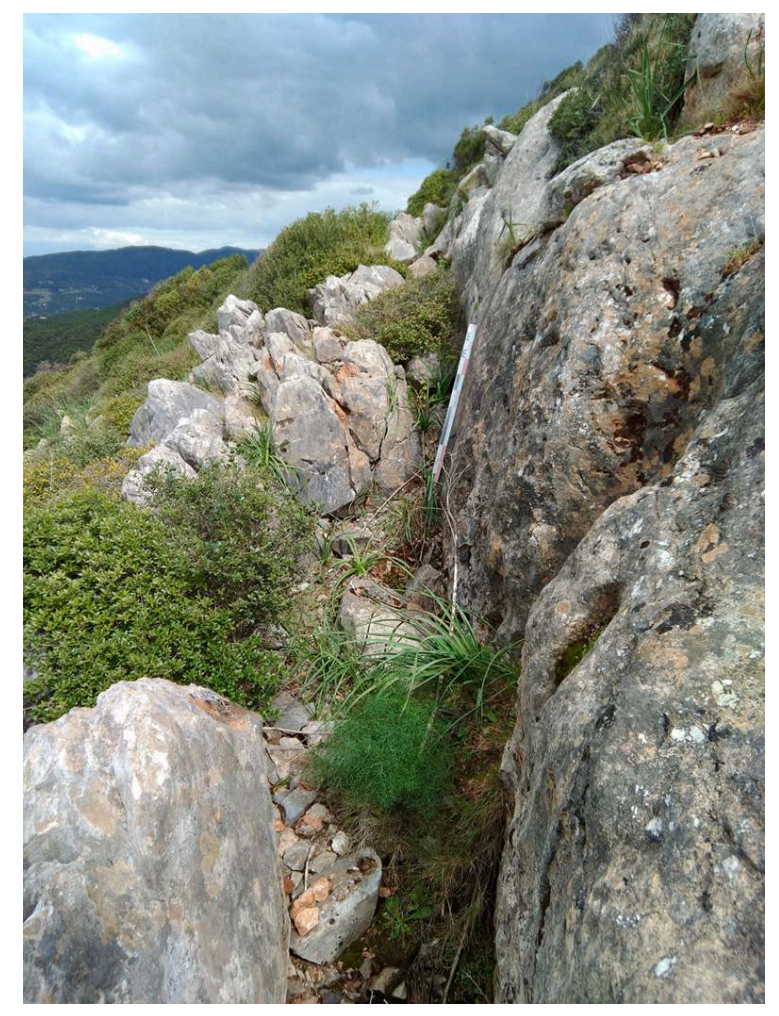

21 Aucun indice archéologique ou documentaire n'était à ce jour attesté sur ces mines. Cela étant, plusieurs fragments de céramiques et d'amphores ont été observés dans les haldes du versant nord-ouest dont de l'amphore italique du $\mathrm{II}^{\mathrm{e}}-\mathrm{I}^{\mathrm{er}} \mathrm{s}$. av. n. è. (Dressel 1) ${ }^{12}$. Il s'agit d'une information notable: les tranchées minières de San Nicolo seraient au moins en partie issues de travaux antiques et il faudra savoir si les travaux du versant oriental appartiennent à cette même phase. Quelques tentatives de reprises contemporaines se trouvent à San Nicolo sans toutefois recouper les ouvrages de surface ni niveler leurs haldes : une galerie de rabais a été ouverte à la base du versant pour atteindre la base des gisements, une autre galerie ouverte à l'extrémité orientale des travaux, et une autre encore ouverte à l'extrémité sud-ouest.

\section{San Giorgio (zone 13)}

Les collines de San Giorgio se trouvent au sud d'Iglesias, entre le Monte Piastu et la Sedda de is fossas. La concentration de vestiges miniers y est considérable: par groupes, les travaux occupent une surface de $1 \mathrm{~km}^{2}$. Sa partie méridionale est composée 
des 4 groupes les plus denses, chacun rassemblant entre 100 et 200 occurrences. Les vestiges sont des puits de 1 à 3 mètres de diamètre et des fosses d'une dizaine de mètres de côté, dont plusieurs donnent accès à de petits réseaux souterrains. Représentés à Is Fossas, il s'agit de travaux en galeries irrégulières, long de quelques dizaines de mètres de long situés à environ $20 \mathrm{~m}$ de profondeur. Plusieurs haldes sont enfin conservées à proximité des fosses. Les vestiges de San Giorgio ont été mis en sécurité dans les années 2000. En préalable, une inspection spéléologique de ces fosses a été réalisée par le Spéléo-club de Domusnovas. À l'issue d'une campagne de topographie, de photographie et d'observation menée en 2002, des choix ont été effectués. Certaines fosses ont été définitivement closes, d'autres conservées en l'état, d'autre enfin ont été identifiées comme exemplaires en cas de valorisation ${ }^{13}$. Le potentiel archéologique est donc encore substantiel.

23 À San Giorgio, aucun indice antique n'a encore été rapporté. De nombreux travaux médiévaux sont en revanche attestés, associés au toponyme médiéval du Monte Barlau, cité dans les archives pisanes. Il s'agit en fait du lieu qui concentre le plus grand nombre de mines mentionnées dans les documents médiévaux : au moins 10 fosses et 4 places pour laver le minerai, situées sur un ruisseau proche. Lors des reconnaissances spéléologiques, le fragment d'un pied de céramique majolique archaïque de pise, datée de la seconde moitié du XIII siècle, a déjà été mis au jour. La question de la datation se pose néanmoins pour les principales fosses. Les gisements ont été certainement exploités à la période contemporaine par des travaux en galerie, mais on ignore s'ils ont commencé en surface en perçant ou ne serait-ce qu'en élargissant ces fosses.

\section{Terras Niedas (zone 14)}

Terras Niedas se situe dans la haute vallée du riu Mannu, sur le versant septentrional du monte Conca s'Ommu. Les vestiges observés rassemblent quelques tranchées à ciel ouvert - la plus grande mesure $20 \mathrm{~m}$ de long sur $7 \mathrm{~m}$ de large - et des puits de 1 à $2 \mathrm{~m}$ de diamètre. Les haldes sont peu ou pas visibles et seules les ruines d'une structure quadrangulaire (4,5 × $2 \mathrm{~m})$ ont été repérées au sommet de la colline.

En ce lieu, aucun indice antique ou mention médiévale ne sont à ce jour connus. Si cette vallée est comprise dans le territoire de la villa d'Antas, citée dans le Bref d'Iglesias avec des mines, alors ces travaux pourraient s'y rattacher. Sur le terrain, aucun indice chronologique probant n'a été relevé. La structure quadrangulaire se trouve à proximité d'une galerie contemporaine, il est donc possible qu'elle soit tardive, mais il faudra le vérifier. Plusieurs travaux à sa base sont issus de reprises contemporaines. Une galerie de rabais notamment semble avoir été creusée pour atteindre la base des gisements. Sa halde marque le versant occidental de la colline.

\section{Perspectives}

À l'issue de cet état des lieux, on dispose d'un corpus archéologique prêt à contribuer aux questionnements de plusieurs périodes et à faire l'objet d'études plus approfondies. À l'échelle de l'Iglesiente, il faudra compléter l'inventaire à chaque fois que de nouvelles informations seront mises au jour, que ce soit dans la documentation ou sur le terrain. Un référentiel des minerais exploités pourra être constitué en partant de matériaux rattachés à ces travaux anciens. À l'échelle de chaque zone, des prospections 
pédestres plus fines pourront être organisées, autant pour améliorer la topographie d'ensemble que pour localiser les lieux de traitement. Le Monte Nuovo et Nanni Frau par exemple seraient des choix pertinents. À l'échelle du réseau minier ou de l'atelier enfin, des vestiges pouvant faire l'objet de fouille et d'étude détaillées sont déjà identifiés : il s'agit notamment du chantier Santa Barbara au Monte Nuovo, de plusieurs puits de San Giorgio et de l'ensemble de la Grotta pisana au Monte San Giovanni avec le bâtiment situé à son entrée.

\section{NOTES}

1. MANCONI (éd.) 1986.

2. BAUDI DI VESME 1870 ; TANGHERONI 1985.

3. Cela pour prolonger plusieurs travaux : MESSINA et al. 2005 ; NASEDDU, PAPINUTO 2005 ; MACRÌ 2012 ; MACRÌ 2016a ; MACRÌ 2016b ; POISSON 2019. Des notices par principales mines sont disponibles en ligne, documentant essentiellement les phases contemporaines : http://www.minieredisardegna.it/. Le Catasto speleologico rassemble aussi plusieurs travaux miniers : https://www.catastospeleologicoregionale.sardegna.it/.

4. L'essentiel des archives contemporaines sont conservées à l'Archivio di Stato de Cagliari, à l'Archivio communale d'Iglesias, à l'Archivio del Distretto minerario d'Iglesias et à l'Archivio storico minerario de Monteponi (IGEA).

5. Fichiers visibles à ces adresses: https://geo-efe.huma-num.fr/layers/ geonode_data:geonode:IGLESIAS_plans_XIX_XX_2021; https://geo-efe.huma-num.fr/ layers/geonode_data:geonode:IGLESIAS_plans_XIX_XX_points_2021.

6. Les zones et les surfaces prospectées sont visibles à ces adresses: https://geoefe.huma-num.fr/layers/geonode_data:geonode:IGLESIAS_Zones_2021; https://geoefe.huma-num.fr/layers/geonode_data:geonode:IGLESIAS_prospections_2021.

7. Fichier visible à cette adresse: https://geo-efe.huma-num.fr/layers/ geonode_data:geonode:IGLESIAS_Releve_2021.

8. La carte rassemblant les couches indiquées plus haut est visible à cette adresse : https://geo-efe.huma-num.fr/maps/20.

9. GIARDINO 1995, p. 140-150.

10. MACRÌ $2016 \mathrm{~b}$, p. 242.

11. Je remercie Angelo Naseddu et les membres du Spéléo-club de Domusnovas pour m'avoir fait visiter la grotta pisana.

12. http://dicocer.cnrs.fr/type/view?indexation=A-ITA_A-ITA+Dr1. Je remercie Benoît Favennec pour cette identification préliminaire.

13. Je remercie Angelo Naseddu, président du Spélo-Club de Domusnovas de m'en avoir informé et de m'avoir communiqué les rapports et documents liés à cette opération. Voir aussi : NASEDDU, PAPINUTO 2005. 


\section{INDEX}

sujets https://ark.frantiq.fr/ark:/26678/pcrtuVBFofOdjD, https://ark.frantiq.fr/ark:/26678/ pcrtcWoSlbWLE0, https://ark.frantiq.fr/ark:/26678/pcrtEpGlZykUTy

Thèmes : EFR

lieux https://ark.frantiq.fr/ark:/26678/pcrtp5hJsnKVpa

chronologie https://ark.frantiq.fr/ark:/26678/pcrtkWqzTusoxz

Année de l'opération : 2021 